referral Triggers Tool would have proactively identified most or all patients who died during their non-elective hospital admission. The inclusion of disease and symptom-related parameters in a PC referral Trigger tool appears feasible as these are often documented in oncology records, whereas oncologists may not routinely identify psychosocial factors.

\section{CREATION OF GUIDELINES FOR MANAGEMENT OF PATIENTS UNDERGOING ABDOMINAL PARACENTESIS FOR MALIGNANT ASCITES AT JOHN EASTWOOD HOSPICE}

Stephanie Hill, Jillian Wall. John Eastwood Hospice

10.1136/bmjspcare-2018-ASPabstracts. 170

Background The inpatient unit at John Eastwood Hospice $(\mathrm{JEH})$ is increasingly providing care for patients with malignant ascites, allowing the relief of symptoms via paracentesis. With no established local or national guidelines to follow for peri procedural care, we felt there were varying approaches used for our patients. We set out to create a local protocol for patients undergoing paracentesis, and audit practice against this.

Methods A thorough literature search alongside review of guidelines uploaded to palliativedrugs.com, and those publicly available on the internet, identified eleven established UK guidelines for management of malignant ascites.

These were used to construct a protocol for use at JEH. Audit standards were then established alongside this, allowing for retrospective analysis of the management of patients who had undergone paracentesis at JEH.

Results 8 patients had undergone this procedure in the preceding 12 months at JEH, giving a total of 10 completed procedures. Peri procedure management in several areas was variable.

A set of baseline observations was documented prior to the procedure in only $10 \%$. Documentation of observations post procedure varied significantly, ranging from half hourly to none at all. $60 \%$ had documentation of drain clamping at some point but in none of the cases was there any further detail of the clamping (e.g. length of time). For the 7 drains which were temporary, the length of time they remained in situ ranged from 31 hours to 195 hours.

Conclusions This retrospective audit demonstrates significant variability in the management of patients undergoing paracentesis at JEH, which strayed from that suggested by the newly created guidelines. These guidelines will now be rolled out in the hospice, alongside education to staff, and re-audit completed to ascertain whether there is more uniform practice.

\section{QUALITATIVE COMPONENT OF A LONGITUDINAL, MIXED METHODS PROGRAMME EVALUATION USING IN-DEPTH INTERVIEWS}

Bridget Johnston, Gillian Mathews, Anne Patterson, Alison Bravington, Beth Hardy, Jane Seymour. University of Glasgow, University of Nottingham, University of York, University of Sheffield

\subsection{6/bmjspcare-2018-ASPabstracts. 171}

Background Macmillan Specialist Care at Home seeks to enhance patient-centred care through community and home- based palliative and end of life care services. This article reports a qualitative study that formed part of a larger evaluation of a multi-site implementation of the specialist palliative care community service.

Aim To examine caregiver, patient and carer experiences of service implementation and identify how its key features: early referral; clinical interventions at home; avoiding hospital admissions; and facilitating patients to die in their preferred place of death, worked in practice.

Methods Qualitative component of a longitudinal, mixed methods programme evaluation using in-depth interviews $(n=49$ [Health professionals $(n=18)$, volunteers $(n=14)$, patients $(n=9)$ and lay carers $(n=8)$ from six community-based sites]) supported by a visual research method 'Pictor' to facilitate dialogue about experiences of care. The data were analysed using thematic analysis.

Results Effective partnerships between generalist and specialist teams improve the overall quality of community and homebased palliative and end of life care. The collaborative approach instils confidence and empowers patients and carers, principal factors in crises-prevention and enabling home deaths. Key themes were:

1. Early referral and rapport; Benefits of seamless care.

2. Averting crises situations;

3. Community consultant as catalyst; Home-based clinical interventions

4. Attending to wider aspects of care; Managing expectations through patient and carer education.

Conclusions Macmillan Specialist Care at Home is a complimentary resource for community-based palliative and end of life care that can extend and greatly enhance the quality of care experience for people with life limiting illnesses and their families. Joint education, training and continuing professional development for specialist teams and existing community staff is advocated to promote shared knowledge and to forge and strengthen bonds between specialist and generalist health and social care professionals. Further testing of the mechanisms involved in implementation will improve transferability potential.

\section{5} IMPLEMENTING EDINBURGH COGNITIVE AND
BEHAVIOURAL ALS SCREEN (ECAS) IN PALLIATIVE CARE
IN PATIENTS WITH MOTOR NEURONE DISEASE (MND) -
EXPERIENCE FROM PHYLLIS TUCKWELL HOSPICE CARE
(PTHC)

Beata LeBon. Phyllis Tuckwell Hospice Care

10.1136/bmispcare-2018-ASPabstracts. 172

Background PTHC has a longstanding interest in neuro-degenerative conditions (NDC) and hosts bi-monthly NDC multidisciplinary team (MDT) meetings on its two sites. Despite other robust care pathways, assessment of cognitive and behavioural function was not standardised. ECAS was identified as the most suitable tool to improve this aspect of care. Methods A 12-month hospice project was designed to:

- Raise awareness of cognitive and behavioural impairment in MND and ECAS 
- Screen all caseload MND patients for suitability to use ECAS and carry out screens. MND Association (MNDA) provided support with a small grant and resources.

All MND patients were assessed during MDT meetings/ other clinical encounters. Offers of the screen (including written information) were made and ECAS was facilitated by the most appropriate clinician.

Results There were 58 MND caseload patients during 1.9.2016-30.10.2017:

- $10(17 \%)$ completed ECAS

- 25 (43\%) were offered ECAS (3 declined; 20 were awaiting decisions/appointments at the time of submission; 1 did not attend the appointment; 1 did not proceed during the appointment)

- $22(38 \%)$ were not considered (2 did not speak English, 7 died before discussion, 13 were too ill/severely cognitively impaired)

ECAS was facilitated by doctors, physiotherapists and occupational therapists in clinics, care homes and homes. Education activities included a dedicated teaching session and dissemination of MNDA booklets and ECAS.

Discussion ECAS worked best in patients with good insight who were motivated to improve their management. The survey is long to complete and dedicated time was need for completion, usually outside regular clinics. Cognitive impairment is a sensitive topic and the introduction of ECAS was most successful following a cue from a patient or after building a trusting rapport before the introductory discussion. Use of ECAS has triggered the hospice collaboration in ongoing national research of the tool.

\section{TRANSFERRING PATIENTS WITH PALLIATIVE CARE NEEDS - WHICH AMBULANCE SERVICE SHOULD YOU USE?}

E Presswood, E O'Brien, N Pease. Wales Deanery, Velindre Cancer Centre, Welsh Ambulance Service Trust

\subsection{6/bmjspcare-2018-ASPabstracts. 173}

Background Transferring patients with palliative care needs between care settings via the Welsh Ambulance Service Trust (WAST) is a daily component of delivering palliative care. We hypothesised that patients would have quicker and more appropriate ambulance transfers if healthcare professionals working in palliative care had a better knowledge of the ambulance services available; including the pilot of the new End-of-Life Rapid Transport Service.

Method We sent an electronic questionnaire to 236 healthcare professionals working in palliative care in Wales. We tested the respondent's knowledge of the available services provided by WAST and asked respondents to share their experiences of transferring patients via the ambulance service.

Results We had 52 responses to the questionnaire (response rate $22 \%$ ). Respondents were predominantly palliative medicine consultants, registrars and clinical nurse specialists. There was a lack of knowledge about the WAST's ability to transfer patients with syringe drivers and the services ability to make decisions regarding not commencing cardio-pulmonary resuscitation. The Urgent Care Service was not well understood and only $12 \%$ of respondents could easily find the correct contact details for each service.
We created and publicised an aide-memoire (attached) about the different ambulance services available in Wales. It contains information tailored to palliative care health care professionals. The aide memoire is designed to be easily accessible in paper and electronic format.

Conclusion Palliative care healthcare professionals lack sufficient knowledge of the ambulance services available in Wales. The aide memoire we created contains the relevant information that is necessary to make the best use of the ambulance services available. The aide memoire is being used across Wales to improve the use of the ambulance service for patients with palliative care needs.

\section{DELIVERING CANCER PATIENT INFORMATION THROUGH SOCIAL MEDIA: WHOSE BENEFIT?}

${ }^{1}$ Nikki Pease, ${ }^{2}$ Simon Noble. 'Velindre Hospital Cardiff, Velindre Cancer Centre; ${ }^{2}$ Cardiff University

10.1136/bmjspcare-2018-ASPabstracts.174

Introduction Historically, access to cancer information has been limited to printed literature and discussions with healthcare professionals. Opportunities through social media and digital technology have seen notable success in adolescent and young adult health care. Its application in the adult cancer population has not been evaluated.

Objectives To identify the extent cancer patients engage with social media and explore its potential for providing access to oncology information.

Method Patients/carers attending a UK cancer centre were surveyed about their access to and use of social media. Its application to their healthcare support needs was explored with specific attention to their information media preferences. Nonpatient identifiable data, based on a prepared questionnaire were collected via hand held tablets and collated via Survey Monkey.

Results 430 participants, comprising 64\% patients and 36\% carers/ spouse, completed the survey. Median age was 65 year old: $82 \%$ were over 50 and $29 \%$ over $70.16 \%$ of patients did not access the Internet whilst $61 \%$ used it daily. Only 13\% used Twitter, 48\% Facebook and 41\% YouTube.

Only $8 \%$ expressed a preference to receive information by digital media, with the majority favouring face-to-face contact with a healthcare professional $(54 \%)$ or printed media (18\%). Talking to other patients or attending patient support groups were the preference of $11 \%$ and $3 \%$ of participant's respectively. $6 \%$ would seek information themselves using a search engine or visiting of government health website.

Conclusion The majority of patients attending a regional cancer are beyond retirement age with less than $18 \%$ under 50 years old. Whilst most have access to the Internet, engagement with Facebook, Twitter and You Tube is uncommon. This suggests a limited role for social

\section{DEVELOPING AN ENHANCED SUPPORTIVE CARE SERVICE FOR WOMEN SUFFERING FROM THE ADVERSE EFFECTS OF CANCER AND ITS TREATMENT}

Katie Taroni, Helen Reeves, Katherine Hill. St. Giles Hospice

10.1136/bmjspcare-2018-ASPabstracts. 175 